\title{
Phase diagram of chirally imbalanced QCD matter
}

\author{
M. N. Chernodub ${ }^{1,2, *}$ and A. S. Nedelin ${ }^{3,4}$ \\ ${ }^{1}$ CNRS, Laboratoire de Mathématiques et Physique Théorique, Université François-Rabelais Tours, \\ Fédération Denis Poisson, Parc de Grandmont, Tours, 37200, France \\ ${ }^{2}$ Department of Physics and Astronomy, University of Gent, Krijgslaan 281, S9, B-9000 Gent, Belgium \\ ${ }^{3}$ Department of Physics and Astronomy, Uppsala University, P. O. Box 803, Uppsala, S-75108, Sweden \\ ${ }^{4}$ Institute for Theoretical and Experimental Physics, B. Cheremushkinskaya 25, Moscow, Russia
}

(Received 4 February 2011; published 11 May 2011)

\begin{abstract}
We compute the QCD phase diagram in the plane of the chiral chemical potential and temperature using the linear sigma model coupled to quarks and to the Polyakov loop. The chiral chemical potential accounts for effects of imbalanced chirality due to QCD sphaleron transitions which may emerge in heavy-ion collisions. We found three effects caused by the chiral chemical potential: the imbalanced chirality (i) tightens the link between deconfinement and chiral phase transitions; (ii) lowers the common critical temperature; (iii) strengthens the order of the phase transition by converting the crossover into the strong first order phase transition passing via the second order end point. Since the fermionic determinant with the chiral chemical potential has no sign problem, the chirally imbalanced QCD matter can be studied in numerical lattice simulations.
\end{abstract}

DOI: 10.1103/PhysRevD.83.105008

PACS numbers: 12.38.Aw, 12.38.Mh, 25.75.Nq

\section{INTRODUCTION}

It is well known that the QCD vacuum has a nontrivial topological structure due to the presence of certain gluon configurations, instantons, which are characterized by an integer-valued topological winding number [1]. For a long time, experimental evidence for the existence of the topological gluon configurations could only be found indirectly, in certain features of the meson spectrum [2].

Recently, it was noticed that a potentially observable direct signature of the topologically nontrivial gluon configurations can emerge in noncentral collisions of heavy ions [3,4]. Such collisions create hot expanding fireballs of the quark-gluon plasma in the background of a strong magnetic field. Topologically nontrivial sphaleron transitions [5,6] can induce-acting via the axial anomaly-a nonvanishing chiral density of quarks in the plasma fireballs. This chirally imbalanced matter is characterized by different densities of right- and left-handed quarks. If such medium is placed in an external magnetic field, then an electric current of quarks should emerge along the field's axis. The unusual generation of the electric current in the magnetic field background is the essence of the so-called "chiral magnetic effect" [3,4]. Signatures of this phenomenon were searched for in heavy-ion experiments at the Brookhaven National Laboratory's Relativistic Heavy Ion Collider (RHIC) [7] and they may probably be observed in the heavy-ion collisions at the Large Hadron Collider (LHC) at CERN.

The chiral magnetic effect is realized in the chirally imbalanced medium in the strong magnetic field background. In order for the chiral magnetic effect to be realized,

*On leave from ITEP, Moscow, Russia. the system of quarks and gluons should be in the deconfined phase, and the chiral symmetry should simultaneously be restored. Both conditions-deconfinement and chiral symmetry restoration - are realized at high temperature. The deconfinement requirement is essential because the system should be able to generate the electric current of individual quarks. Moreover, in the chirally broken (low temperature) phase, the chiral imbalance should quickly be washed out due to the presence of the chiral condensate which facilitates transitions between left-handed and right-handed quarks. Therefore, it becomes interesting to investigate the influence of the background magnetic field and the effect of the chiral imbalance on the finite-temperature transition between hadron and quark-gluon plasma phases.

The effect of the strong magnetic field on the QCD phase transition has been studied both analytically [8-11] and numerically [12]. All these studies have found that the magnetic field background increases the transition temperature and makes the phase transition stronger. A third effect was possibly found in Ref. [10]: the magnetic field may split the deconfining and chiral phase transitions, thus leading to emergence of the new, chirally broken deconfining phase. (The splitting can be small [11], however.) In the low-temperature and strong-magnetic-field corner of the QCD phase diagram, a new electromagetically superconducting phase may emerge [13].

The topologically induced changes in chirality can be modeled with the help of the chiral chemical potential $\mu_{5}$ which creates a difference between the right- and lefthanded particles [4]. This potential can be related to the $\theta$ angle of strong interactions as follows [4]:

$$
\mu_{5}=\frac{\partial}{\partial t} \frac{\theta}{2 N_{f}}
$$


where $t$ is the time coordinate and $N_{f}$ is the number of the light flavors in the theory.

The influence of the chiral imbalance on the thermal phase transition in the magnetic field background was addressed in Ref. [9] regarding possible applications to the chiral magnetic effect. Working in the Nambu-JonaLasinio (NJL) model coupled to the Polyakov loop (PNJL), the authors of Ref. [9] have found that the chiral imbalance makes the temperature of the chiral phase transition smaller while the strength of the transition becomes stronger. In our paper we confirm the findings of Ref. [9] working at zero magnetic field in the linear sigma model coupled to quarks and to the Polyakov loop $\left(\operatorname{PLSM}_{q}\right)$ which also serves as an effective low-energy model of QCD. Our main result is the QCD phase diagram in the $\left(\mu_{5}, T\right)$ plane, which is plotted in Fig. 4.

The structure of this paper is as follows: In Sec. II, we describe our model $\left(\mathrm{PLSM}_{q}\right)$. In Sec. III, we discuss thermodynamics and calculate the phase diagram of the model. Section IV is devoted to our conclusions.

\section{THE MODEL}

We use a linear sigma model coupled to quarks [14] and to the Polyakov loop $\left(\mathrm{PLSM}_{q}\right)$ [15]. The inclusion of the Polyakov loop allows us to account for effect of the color confinement following the similar proposal in the NJL model $[16,17]$.

This low-energy model of QCD contains three types of fields: the doublet of the quark fields $\psi(x)=(u, d)^{T}$, the scalar chiral (meson) fields $(\sigma, \vec{\pi})$ with $\vec{\pi}=\left(\pi_{1}, \pi_{2}, \pi_{3}\right)$, and the complex-valued scalar field of the Polyakov loop:

$$
\begin{gathered}
\Phi(x)=\frac{1}{3} \operatorname{Tr} L(x), \quad \bar{\Phi}(x)=\frac{1}{3} \operatorname{Tr} L^{\dagger}(x), \\
L=\mathcal{P} \exp \left[i \int_{0}^{1 / T} d \tau A_{4}(\vec{x}, \tau)\right]
\end{gathered}
$$

where $A_{4}=i A_{0}$ is a rescaled timelike component of the $S U(3)$ gauge field, $\mathcal{P}$ is the path-ordering operator, $\vec{\pi}$ is the isotriplet of the pseudoscalar pions, and $\sigma$ is the pseudoscalar field.

The $\operatorname{PLSM}_{q}$ Lagrangian can be represented as a sum of the following three parts:

$$
\mathcal{L}=\mathcal{L}_{q}(\bar{\psi}, \psi, \sigma, \vec{\pi}, \Phi, \bar{\Phi})+\mathcal{L}_{\sigma}(\sigma, \vec{\pi})+\mathcal{L}_{\Phi}(\Phi, \bar{\Phi}) .
$$

The quark part of the Lagrangian (3),

$$
\mathcal{L}_{q}=\bar{\psi}\left[i \not D-g\left(\sigma+i \gamma^{5} \vec{\tau} \cdot \vec{\pi}\right)+\mu_{5} \gamma^{0} \gamma^{5}\right] \psi,
$$

provides the interaction between the quarks $\psi$, the chiral fields $\sigma, \vec{\pi}$, and the field $A_{\mu}$ via the covariant derivative $\not D=\gamma^{\mu}\left(\partial_{\mu}-i A_{\mu}\right)$. The Lagrangian (4) also includes the real-valued chiral chemical potential $\mu_{5}$.

The dynamics of the chiral fields is described by the second term in the Lagrangian (3),

$$
\begin{aligned}
\mathcal{L}_{\sigma}(\sigma, \vec{\pi})= & \frac{1}{2}\left(\partial_{\mu} \sigma \partial^{\mu} \sigma+\partial_{\mu} \pi^{0} \partial^{\mu} \pi^{0}\right) \\
& +\partial_{\mu} \pi^{+} \partial_{\mu} \pi^{-}-V_{\sigma}(\sigma, \vec{\pi}),
\end{aligned}
$$

where we have introduced charged and neutral mesons,

$$
\pi^{ \pm}=\frac{1}{\sqrt{2}}\left(\pi^{1} \mp i \pi^{2}\right), \quad \pi^{0}=\pi^{3},
$$

respectively. The first and the second terms of the potential,

$$
V_{\sigma}(\sigma, \vec{\pi})=\frac{\lambda}{4}\left(\sigma^{2}+\vec{\pi}^{2}-v^{2}\right)^{2}-h \sigma,
$$

provide, respectively, (strong) spontaneous and (weak) explicit breaking of the chiral symmetry. The phenomenologically acceptable parameters in Eqs. (4) and (7) are: $g=3.3, \lambda=20$, and $v=87.7 \mathrm{MeV}$ [18]. We work in a mean field approximation, thus neglecting quantum fluctuations (i.e., the kinetic terms) of the scalar fields $\sigma$ and $\vec{\pi}$.

Effects of the color confinement are encoded in the last term of the Lagrangian (3), which describes the potential of the Polyakov loop:

$$
\mathcal{L}_{\Phi}=-V_{\Phi}(\Phi, \bar{\Phi}, T) .
$$

As in the previous case involving the mesonic chiral fields, we neglect possible kinetic terms for the Polyakov loop and consider only the potential term, following Ref. [19]:

$$
\begin{aligned}
\frac{V_{\Phi}(\Phi, \bar{\Phi}, T)}{T^{4}}= & -\frac{1}{2} a(T) \bar{\Phi} \Phi+b(T) \\
& \times \ln \left[1-6 \bar{\Phi} \Phi+4\left(\bar{\Phi}^{3}+\Phi^{3}\right)-3(\bar{\Phi} \Phi)^{2}\right],
\end{aligned}
$$

where

$$
\begin{aligned}
& a(T)=a_{0}+a_{1}\left(\frac{T_{0}}{T}\right)+a_{2}\left(\frac{T_{0}}{T}\right)^{2}, \\
& b(T)=b_{3}\left(\frac{T_{0}}{T}\right)^{3},
\end{aligned}
$$

and $T_{0}=270 \mathrm{MeV}$ is the temperature of the deconfinement phase transition in the pure $S U(3)$ Yang-Mills theory without quarks [20]. Another choice of the critical temperature can be made by rescaling the parameter $T_{0}$ from 270 to $190 \mathrm{MeV}$ in order to make a closer match with available lattice data [17]. The coefficients in Eq. (10) are:

$$
\begin{array}{ll}
a_{0}=16 \pi^{2} / 45 \approx 3.51, & a_{1}=-2.47, \\
a_{2}=15.2, & b_{3}=-1.75 .
\end{array}
$$

The Polyakov loop potential (9) respects the center $\mathbb{Z}_{3}$ symmetry, $\Phi \rightarrow e^{2 \pi n i / 3} \Phi$ with $n=0,1,2$.

\section{THERMODYNAMICS AND PHASE DIAGRAM}

The thermodynamic potential per unit volume is:

$$
\Omega=-\frac{T}{V_{3 d}} \ln Z,
$$


where $Z$ is the partition function and $V_{3 d}$ is the volume of the three-dimensional space. In the mean field approximation, the chiral fields and Polyakov loop field are considered as classical objects, so that the thermodynamic potential can be rewritten as

$$
\begin{aligned}
\Omega\left(\sigma, \vec{\pi}, L, \Phi, \bar{\Phi}, \mu_{5}\right)= & V_{\phi}(\sigma, \vec{\pi})+V_{\Phi}(\Phi, \bar{\Phi}, T) \\
& +\Omega_{q}\left(\sigma, \vec{\pi}, \Phi, \bar{\Phi}, T, \mu_{5}\right),
\end{aligned}
$$

where the potential $V_{\phi}$ is given by Eq. (7) and the potential $V_{\Phi}$ is described in Eqs. (9)-(11). In Eq. (13), the quark part $\Omega_{q}$ comes from the fermion determinant, which can be computed by explicit diagonalization of the quadratic operator corresponding to the quark Lagrangian (4):

$$
\begin{aligned}
\Omega_{q}= & -2 \sum_{s= \pm 1} \int \frac{d^{3} p}{(2 \pi)^{3}}\left(3 \omega_{s}+T\left\{\operatorname { l n } \left[1+3\left(\bar{\Phi}+\Phi e^{-\omega_{s} / T}\right)\right.\right.\right. \\
& \left.\left.\left.\times e^{-\omega_{s} / T}+e^{-3 \omega_{s} / T}\right]+ \text { c.c. }\right\}\right)
\end{aligned}
$$

where $\Phi$ is the Polyakov loop (2) and the fermion spectrum is

$$
\omega_{s}(p)=\sqrt{\left(|p| s-\mu_{5}\right)^{2}+g^{2}\left(\sigma^{2}+\vec{\pi}^{2}\right)} .
$$

Here $s= \pm 1$ is the helicity (the sign of the projection of the particle's spin on the direction of the particle's motion). The first term in Eq. (14) corresponds to the divergent energy of the Dirac sea. After a proper regularization, the contribution from this term renormalizes the parameters $\lambda$ and $v$ of the chiral potential (7) also adding finite logarithmic corrections (which are sometimes called zero-point corrections) to the potential. These corrections have a pure vacuum origin because they depend on neither temperature nor the chiral chemical potential. Usually, these logarithmic corrections are disregarded in the phenomenological approaches based on the linear sigma model because their contribution does not change the qualitative physical picture. We ignore the logarithmic corrections as well, referring the interested reader to studies of the vacuum fluctuations effects done in Refs. [21,22].

Integrating Eq. (14) by parts, we can rewrite the thermodynamic potential in the following form:

$$
\Omega_{q}=-\frac{1}{3 \pi^{2}} \sum_{s= \pm 1} \int_{0}^{\infty} d p p^{3}\left[n_{q, s}(p)+n_{\bar{q}, s}(p)\right] \frac{\partial \omega_{s}(p)}{\partial p},
$$

where $n_{q, s}$ and $n_{\bar{q}, s} \equiv n_{q, s}^{*}$ are, respectively, the real-valued occupation numbers (summed over colors) for quarks and antiquarks carrying the helicity $s$, and

$$
n_{q, s}=\frac{3 e^{-\omega_{s} / T}\left(\Phi+2 \bar{\Phi} e^{-\omega_{s} / T}+e^{-2 \omega_{s} / T}\right)}{1+3\left(\Phi+\bar{\Phi} e^{-\omega_{s} / T}\right) e^{-\omega_{s} / T}+e^{-3 \omega_{s} / T}} .
$$

The mean field values of the fields $\sigma, \vec{\pi}$, and $\Phi$ are found by a (numerical) minimization of the thermodynamic potential given by Eqs. (13), (7), (9)-(11), and (16) with respect to the variations of $\sigma, \vec{\pi}$, $\Phi$, and $\bar{\Phi}$, Eq. (2), at fixed values of the temperature $T$ and chiral chemical potential $\mu_{5}$. The minimum of the thermodynamic potential is always reached at the real values of the (traced) Polyakov loop $\Phi$. We found that, in the presence of the chiral chemical potential, $\mu_{5} \neq 0$ (and in the absence of the baryon chemical potential $\mu$ ), the minimum is always reached at $\Phi=\bar{\Phi}$, contrary to the $\mu \neq 0$ case.

The fermion determinant breaks the center $\mathbb{Z}_{3}$ symmetry of the Polyakov loop potential, and the global minimum of the thermodynamic potential (13) corresponds to the realvalued Polyakov loop. Moreover, one can show numerically that the presence of the charged pion condensates $\pi^{ \pm}$ makes the thermodynamic potential larger so that these condensates are disfavored, $\left\langle\pi^{ \pm}\right\rangle=0$. The last term in Eq. (7) forces the neutral pion condensate to be zero, $\left\langle\pi^{0}\right\rangle=0$.

Therefore we are left with two unknown expectation values which are to be fixed by the minimization of the thermodynamic potential. These are the real part of the Polyakov loop, $\operatorname{Re} \Phi$, and the chiral order parameter $\sigma$.

The expectation values of the Polyakov loop and of the chiral order parameter $\sigma$ are shown as functions of the temperature in Figs. 1 and 2, respectively. The chiral field is normalized to unity at $T=\mu_{5}=0$, and the corresponding vacuum expectation value $\sigma_{0}$ is given by the pion decay constant:

$$
\sigma_{0} \equiv\langle\sigma\rangle_{T=\mu_{5}=0} \equiv f_{\pi}\left(T=\mu_{5}=0\right)=92.2 \mathrm{MeV} .
$$

The temperature and chiral chemical potential in Figs. 1 and 2 are expressed in units of the critical transition temperature $T_{c}^{(0)}$ at zero chiral chemical potential in $\mathrm{PLSM}_{q}$ :

$$
T_{c}^{(0)} \equiv T_{c}\left(\mu_{5}=0\right)=213.2 \mathrm{MeV} \quad\left[\text { in } \operatorname{PLSM}_{q}\right]
$$

Because of the presence of the explicit symmetrybreaking term in the chiral potential $V_{\sigma}$, Eq. (7), the transition between the quark-gluon plasma phase and the

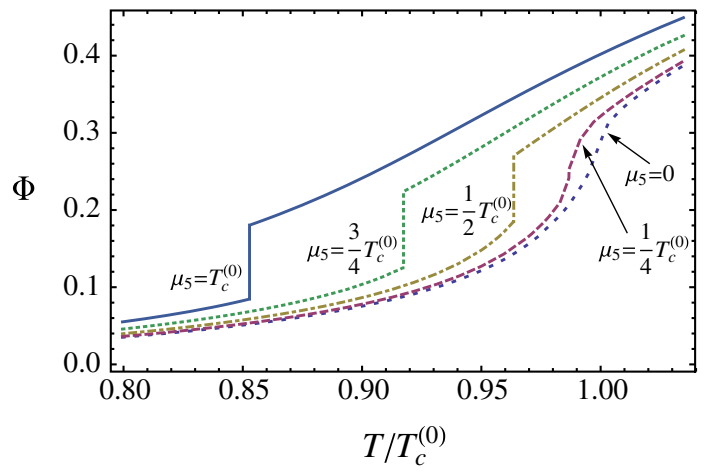

FIG. 1 (color online). The expectation value of the Polyakov loop $\Phi \equiv \bar{\Phi}$ as the function of temperature $T$ at fixed values of the chiral chemical potential $\mu_{5} . T$ and $\mu_{5}$ are given in units of $T_{c}^{(0)}$, the critical temperature of the $\mu_{5}=0$ transition, Eq. (19). 


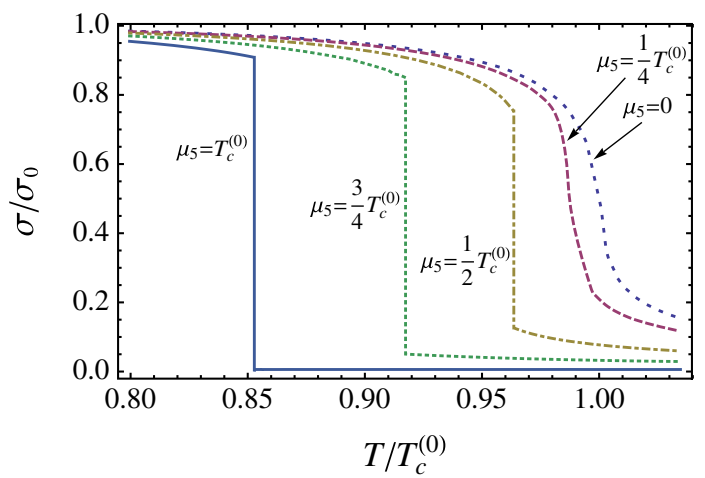

FIG. 2 (color online). The chiral order parameter $\sigma$. We use the same notations as in Fig. 1. The value of $\sigma_{0}$ is given in Eq. (18).

hadron phase at zero chemical potential is a smooth crossover. The critical temperature value in Eq. (19) corresponds to the temperature where the slopes of the Polyakov loop $\Phi$ and the chiral field $\sigma$ are steepest. (The difference in the critical values for these two quantities is smaller than $1 \mathrm{MeV}$ in $\operatorname{PLSM}_{q}$.)

According to Figs. 1 and 2, the crossover turns into the first order transition as the chiral chemical potential $\mu_{5}$ increases. (The larger $\mu_{5}$, the stronger the transition.) The critical temperature of the transition between the quarkgluon plasma phase and the hadron phase decreases as the chiral chemical potential increases. Moreover, the chiral and deconfinement phase transitions are tightened to each other, and they do not split as the value of $\mu_{5}$ increases.

Obviously, the presence of the nonzero chiral chemical potential $\mu_{5}$ does not induce a nonvanishing baryon charge, so that the quark density $n$ is always zero,

$$
n=2 \sum_{s= \pm 1} \int \frac{d^{3} p}{(2 \pi)^{3}}\left[n_{q, s}(p)-n_{\bar{q}, s}(p)\right] \equiv 0 .
$$

However, the density of the chiral charge (i.e., the difference between the densities of the right- and left-handed particles),

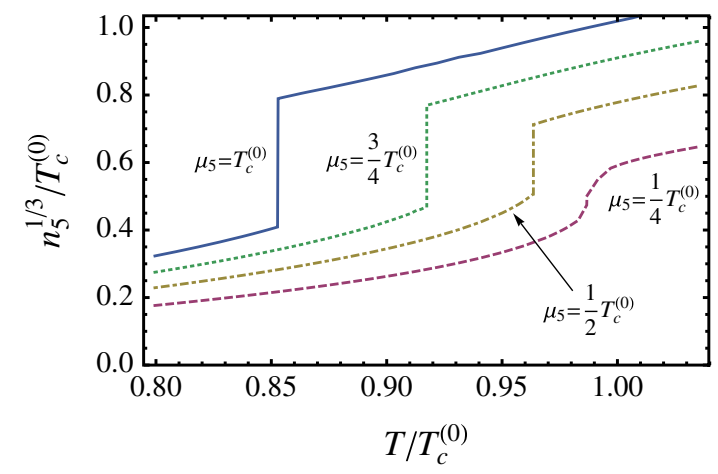

FIG. 3 (color online). The one-third power of the chiral charge density $n_{5}$, Eq. (21), as a function of temperature $T$ at a fixed set of the chiral chemical potential $\mu_{5}$. We use the same units as in Fig. 1.

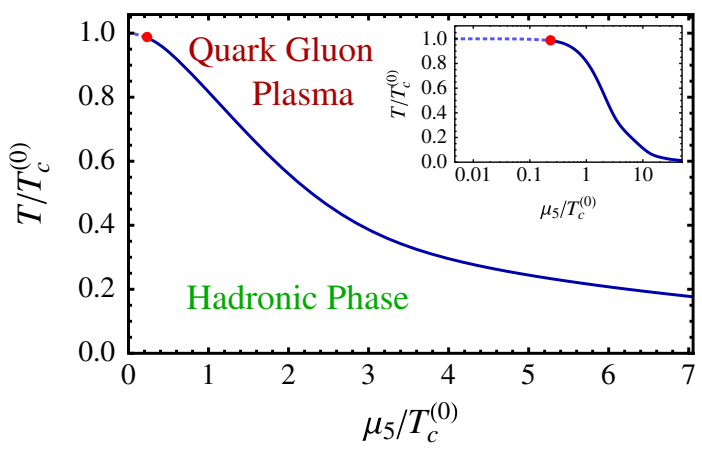

FIG. 4 (color online). Phase diagram of QCD in the plane of the chiral chemical potential $\mu_{5}$ and temperature $T$, expressed in units of the critical transition temperature $T_{c}^{(0)}$ at $\mu_{5}=0$, Eq. (19). The dashed line to the left of the [red] point represents the smooth crossover; the solid line corresponds to the first order phase transition; and the [red] point marks the second order critical end point (22). The inset shows the same phase diagram with the chiral chemical potential axis plotted in the logarithmic scale.

$$
n_{5}=n_{R}-n_{L}=\frac{\left\langle N_{5}\right\rangle}{V_{3 d}}=\left\langle\bar{\psi} \gamma^{0} \gamma^{5} \psi\right\rangle=-\frac{\partial \Omega_{q}}{\partial \mu_{5}},
$$

should in general be nonzero at a nonvanishing chiral chemical potential (and, naturally, $n_{5}=0$ at $\mu_{5}=0$ ).

We plot (the one-third power of) the density of the chiral charge (21) in Fig. 3. The chiral imbalance of the media in the presence of a fixed chemical potential $\mu_{5}$ is a growing function of the temperature. The chiral charge density $n_{5}$ is strongly enhanced at the transition temperature $T_{c}=$ $T_{c}\left(\mu_{5}\right)$, as the system goes from the hadron phase to the quark-gluon plasma phase.

The phase diagram in the $\left(\mu_{5}, T\right)$ plane is shown in Fig. 4.

The transformation of the crossover transition (the dashed line at lower values of $\mu_{5}$ ) to the first order phase transition (the solid line at larger $\mu_{5}$ ) goes via a critical end point (CEP) in which the transition becomes a second order transition. We found that the CEP (the [red] point in Fig. 4) is located at

$$
\left(\mu_{5}, T\right)^{\mathrm{CEP}}=\left(0.232 T_{c}^{(0)}, 0.998 T_{c}^{(0)}\right) .
$$

Our phase diagram, Fig. 4, computed in the $\mathrm{PLSM}_{q}$ at zero magnetic field agrees qualitatively with the corresponding phase diagram of Ref. [9], where the calculations are done in the PNJL model in a weak magnetic field background $^{1}$ in the window $0 \leq \mu_{5} \lesssim 2.3 T_{c}^{(0)}$. The critical curves $T_{c}=T_{c}\left(\mu_{5}\right)$ in the $\operatorname{PLSM}_{q}$ and PNJL models are quite close to each other, while the positions of the corresponding CEPs differ from each other substantially, given that the location of the CEP in the PNJL model is

\footnotetext{
${ }^{1}$ The critical temperature curve $T_{c}=T_{c}\left(\mu_{5}, B\right)$ is almost independent of the strength of the magnetic field $B$ in a weak field [9].
} 
$\left(\mu_{5}, T\right)^{\mathrm{CEP}}=\left(1.7 T_{c}^{(0)}, 0.8 T_{c}^{(0)}\right)$ according to Ref. [9]. The difference between the PNJL prediction and our result (22) can be attributed either to the model-dependent issues or to the fact that we have neglected the vacuum corrections coming from the fermionic determinant which may influence the $\operatorname{PLSM}_{q}$ thermodynamics quantitatively [22].

It is important to mention that the line of the first order phase transition does not hit the $\mu_{5}$ axis at any finite value of the chiral chemical potential. Instead, the $\operatorname{PLSM}_{q}$ model predicts that the $T_{c}=T_{c}\left(\mu_{5}\right)$ curve approaches this axis smoothly at asymptotically large values of the chiral chemical potential $\mu_{5}$ :

$$
\lim _{\mu_{5} \rightarrow \infty} T_{c}\left(\mu_{5}\right)=0 .
$$

Notice, however, that $\mathrm{PLSM}_{q}$ is an effective low-energy model of QCD, and therefore, at large values of massive parameters (for example, at large $\mu_{5}$ ), the results coming from this model may become inaccurate. Therefore, the prediction in Eq. (23) should be considered with care.

\section{CONCLUSIONS}

The chirally imbalanced hot quark-gluon plasma may emerge in heavy-ion collisions at RHIC and LHC experimental facilities. We have computed the QCD phase diagram in the chirally imbalanced background at finite temperature (Fig. 4) using the linear sigma model coupled to quarks and to the Polyakov loop $\left(\mathrm{PLSM}_{q}\right)$ at zero magnetic field. Our results are in qualitative agreement with conclusions of an earlier study of the QCD finitetemperature phase transition in the PNJL model in simultaneously imposed chiral and magnetic field backgrounds [9].

We have found that the increase of the chiral chemical potential $\mu_{5}$ tightens the link between deconfinement and chiral phase transitions, simultaneously lowering the common critical temperature and strengthening the order of the phase transition by converting the crossover (realized at low chiral imbalance) into the first order phase transition (found at higher chiral imbalance). The location of the second-order critical end point-at which the crossover turns into the first order phase transition-is given in Eq. (22). The $\operatorname{PLSM}_{q}$ predicts that, at strictly zero temperature, the system always stays in the hadronic phase regardless of the value of the chiral chemical potential [Eq. (23) and Fig. 4].

We are mainly interested in the finite-temperature phase diagram of QCD at nonzero chiral chemical potential because the chiral magnetic effect is realized in the presence of the strong magnetic field in the chirally imbalanced background [4]. We confirm that some effects of the strong magnetic field and the chiral chemical potential on the critical temperature of the QCD phase transition are opposite: the magnetic field tends to increase the temperature of the phase transition [8-12] while the chirally imbalanced background forces the critical temperature to become lower [9]. Moreover, the magnetic field background splits the chiral and deconfinement phase transitions [10] (although the splitting can be small [11]), while the chiral imbalance tightens the link between these transitions. However, despite these dissimilarities, the external magnetic field and the chiral imbalance have one common feature: they both make the QCD phase transition stronger.

Finally, we would like to note that the predictions for the QCD phase diagram in the $\left(\mu_{5}, T\right)$ plane, given in Fig. 4 of the present article and in Ref. [9], can be directly tested from the first principles in numerical simulations of lattice QCD because the fermionic determinant with the chiral chemical potential has no sign problem [4].

\section{ACKNOWLEDGMENTS}

The authors are very grateful to M. Ruggieri for interesting discussions and useful comments. This work was partially supported by Grant No. ANR-10-JCJC-0408 HYPERMAG (France) and by STINT Institutional Grant No. IG2004-2 025 (Sweden).
[1] A. A. Belavin, A. M. Polyakov, A. S. Schwartz, and Yu. S. Tyupkin, Phys. Lett. 59B, 85 (1975).

[2] E. Witten, Nucl. Phys. B156, 269 (1979); G. Veneziano, Nucl. Phys. B159, 213 (1979).

[3] D. Kharzeev, Phys. Lett. B 633, 260 (2006).

[4] K. Fukushima, D. E. Kharzeev, and H. J. Warringa, Phys. Rev. D 78, 074033 (2008).

[5] N. S. Manton, Phys. Rev. D 28, 2019 (1983); F. R. Klinkhamer and N.S. Manton, Phys. Rev. D 30, 2212 (1984).

[6] G. D. Moore, in Strong and Electroweak Matter 2000: Proceedings of the SEWM2000 Meeting, Marseille,
France, 13-17 June 2000 (World Scientific, Singapore, 2001), arXiv:hep-ph/0009161.

[7] B. I. Abelev et al. (STAR Collaboration), Phys. Rev. Lett. 103, 251601 (2009); Phys. Rev. C 81, 054908 (2010).

[8] E. S. Fraga and A.J. Mizher, Phys. Rev. D 78, 025016 (2008).

[9] K. Fukushima, M. Ruggieri, and R. Gatto, Phys. Rev. D 81, 114031 (2010).

[10] A. J. Mizher, M. N. Chernodub, and E. S. Fraga, Phys. Rev. D 82, 105016 (2010); R. Gatto and M. Ruggieri, Phys. Rev. D 82, 054027 (2010). 
[11] R. Gatto and M. Ruggieri, Phys. Rev. D 83, 034016 (2011).

[12] M. D'Elia, S. Mukherjee, and F. Sanfilippo, Phys. Rev. D 82, 051501 (2010).

[13] M. N. Chernodub, Phys. Rev. D 82, 085011 (2010); Phys. Rev. Lett. 106, 142003 (2011).

[14] M. Gell-Mann and M. Lévy, Nuovo Cimento 16, 705 (1960).

[15] E. Megias, E. R. Arriola, and L. L. Salcedo, Phys. Rev. D 74, 065005 (2006).

[16] K. Fukushima, Phys. Lett. B 591, 277 (2004).

[17] C. Ratti, M. A. Thaler, and W. Weise, Phys. Rev. D 73, 014019 (2006).
[18] O. Scavenius, A. Mocsy, I. N. Mishustin, and D. H. Rischke, Phys. Rev. C 64, 045202 (2001).

[19] S. Roessner, T. Hell, C. Ratti, and W. Weise, Nucl. Phys. A814, 118 (2008).

[20] F. Karsch, E. Laermann, and A. Peikert, Nucl. Phys. B605, 579 (2001).

[21] A. Mocsy, I. N. Mishustin, and P. J. Ellis, Phys. Rev. C 70, 015204 (2004); J. K. Boomsma, and D. Boer, Phys. Rev. D 80, 034019 (2009); T. K. Herbst, J. M. Pawlowski, and B. J. Schaefer, Phys. Lett. B 696, 58 (2011).

[22] V. Skokov, B. Stokic, B. Friman, and K. Redlich, Phys. Rev. C 82, 015206 (2010). 